\title{
Effects of a Time Varying Moment on a Large Tethered Satellite in Circular or Elliptical Orbit
}

\author{
Narayan R. Iyer ${ }^{1}$ \\ 701 W Stadium Avenue, West Lafayette, Indiana 47907; nriyer2@gmail.com
}

\begin{abstract}
The purpose of this paper is to investigate the effect of a varying moment on the rotation angle of a large tethered satellite that is orbiting a planet. Two different types of orbits were investigated: a simple circular orbit and an elliptical orbit. Cases with zero and non-zero initial angular rotation velocity were investigated as well. This investigation will assist satellite docking missions. The large rigid tethered satellite is a futuristic concept, and this investigation is meant to assist possible docking missions to the satellite. To simplify the problem, the rotation is constrained to the orbital plane.
\end{abstract}

${ }^{1}$ Current Address: 515 2nd Ave SW, Apt 105, Cedar Rapids, IA 52404 


\section{Introduction}

In the futuristic case of a very large tethered satellite in orbit around a body, different parts of the satellite face different gravitational accelerations, causing the center of gravity to deviate from the center of mass and vary with time. This causes varying torques on the satellite. The satellite discussed in this paper is a very long system consisting of two stations and one long rigid tether connecting them. Figure 1 below conveys the satellite system.

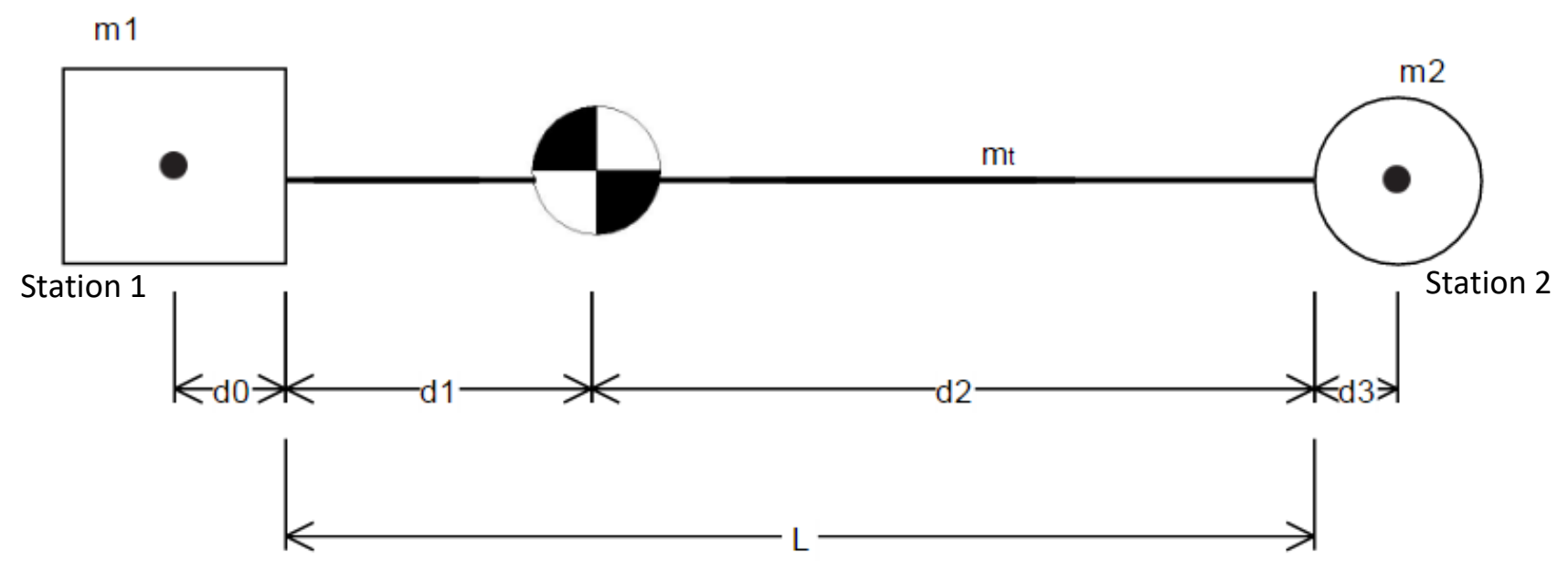

Figure 1. Large tethered satellite. The figure conveys the large tethered satellite. L is the total length of the tether. The CM symbol conveys the center of mass NOT center of gravity. The location of the center of gravity will not be discussed in this paper. mt is the mass of the tether. 


\section{Methods}

The initial conditions to the investigation will define $\mathrm{m} 1, \mathrm{~m} 2, \mathrm{~d} 0$ and $\mathrm{d} 3 \mathrm{~d} 1$ and $\mathrm{d} 2{ }^{2}$ will be found as a first step to the investigation using the equation below. The center of mass is calculated from center of station one.

$$
R_{c m} M_{\text {satellite }}=\left(d 0+\frac{L}{2}\right) m_{t}+(d 0+L+d 3) m_{2}
$$

Where $R_{c m}=(d 0+d 1)$, and $M_{\text {satellite }}=m_{1}+m_{2}+m_{t}$

The only unknown in this equation is $d 1$ because all the other terms in the equation will be defined in the initial conditions of the investigation. Therefore rearranging equation 1 to solve for $d 1$, we get:

$$
d_{1}=\left((d 0+L / 2) m_{t}+(d 0+L+d 3) m_{2}-M_{\text {satellite }} d 0\right) / M_{\text {satellite }}
$$

The center of mass will be the axis of rotation since the moment of inertia is minimized at that point. The diagrams below convey the satellite in circular and elliptical orbits.

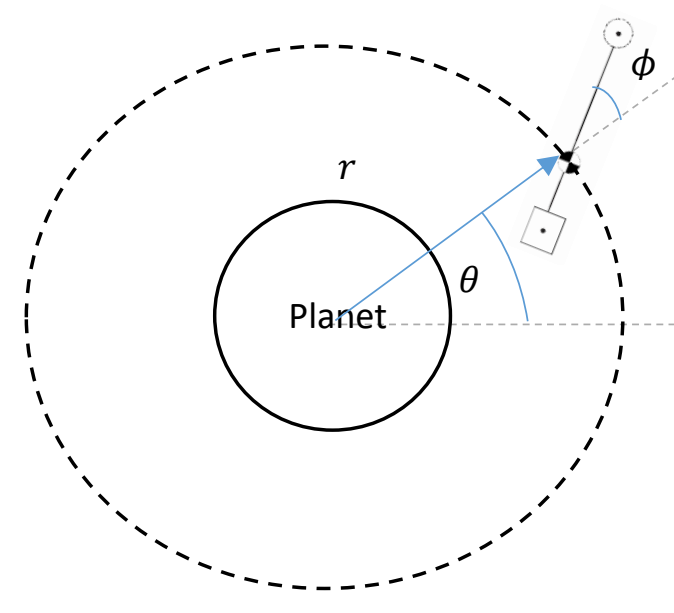

Figure 2. Tethered Satellite in Circular Orbit. The thick dashed line traces out the locus of the center of mass along the orbit.

\footnotetext{
${ }^{2}$ See Nomenclature at end of paper
} 


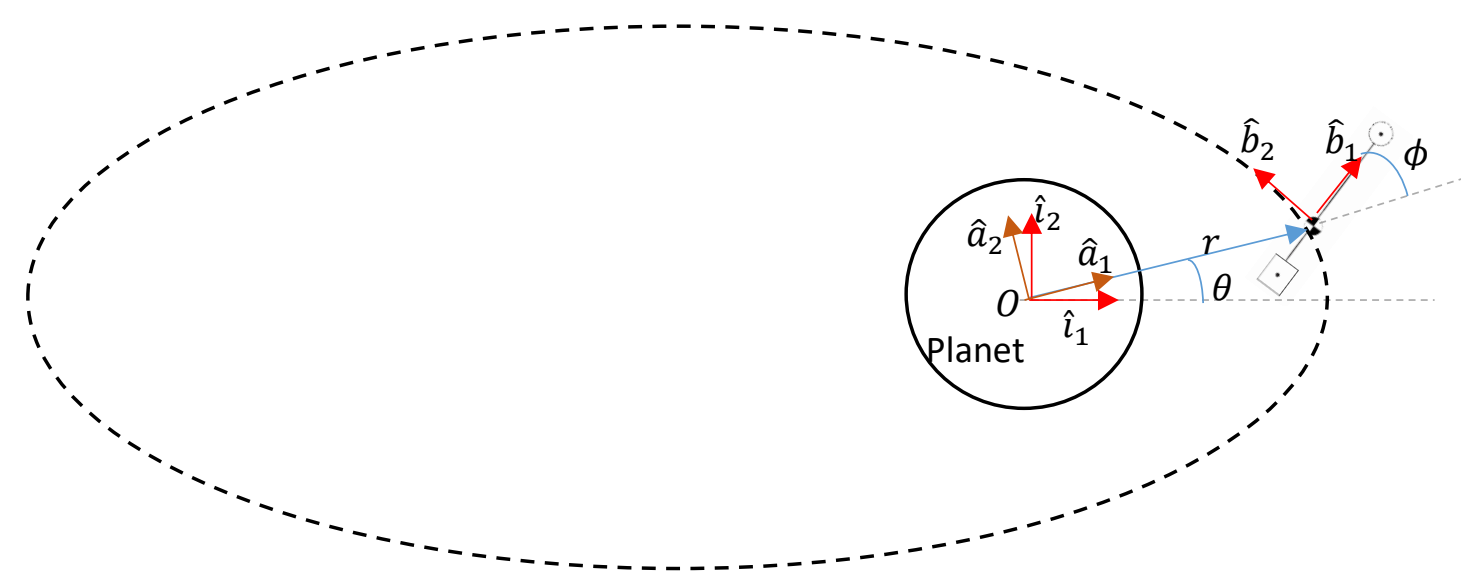

Figure 3. Tethered Satellite in Elliptical Orbit. The dashed line traces out the locus of the center of mass along the orbit. The unit vectors representing different reference frames are also conveyed in this figure. The same unit vectors apply to the circular orbit as well.

\subsection{Test Case Introduction}

First, an equation of motion was found for the circular orbit scenario. The single equation of motion will describe the behavior of the unknown variable that is $\phi$, the angle of rotation of the satellite. Then the state space representation was performed for the equation of motion for use in the ode45 program. Numerical results were then obtained for several cases. The cases investigated for the circular orbit are given in the list below:

3.1.1 Zero initial rotational angular velocity $(\dot{\phi}(0)=0$ degrees per second)

1) $\phi(0)=0$ degrees, non-symmetric satellite

2) $\phi(0)=30$ degrees, non-symmetric satellite

3) $\phi(0)=60$ degrees, non-symmetric satellite

4) $\phi(0)=90$ degrees, symmetric satellite

5) $\phi(0)=90$ degrees, non-symmetric satellite

3.1.2 Small initial rotational angular velocity $(\dot{\phi}(0) \neq 0)$

6) $\dot{\phi}(0)=0.01$ degree/second, $\phi(0)=60$ degrees, Asymmetric satellite

7) $\dot{\phi}(0)=0.1$ degree/second, $\phi(0)=60$ degrees, Asymmetric satellite

For each of the above cases, the rotational angle time history will be found along with the net moment and angular velocity time histories. 
Next for the elliptical orbit, the same equation of motion can be used with several changes. The radius of orbit will vary with the orbit angle from periapsis, $\theta$. However, elliptical orbit equations cannot be manipulated to convey $\theta$ explicitly as a function of time. Therefore, a process to solve the for the radius of orbit is discussed within the paper, and a numerical solution was found for the elliptical orbit of the rotating tethered satellite. 


\section{Theory and Calculations}

Euler's equation of motion relates the torque faced by the satellite to its moment of inertia and its angular acceleration. Using the Euler's equation of motion (with x pointing out of the page) ${ }^{3}$ :

$$
M_{x}=I_{x} \dot{\omega}_{x}+\left(I_{Z}-I_{Y}\right) \omega_{Y} \omega_{Z}
$$

Since the rotation occurs at a single plane, $\omega_{Y}$ and $\omega_{Z}$ are zero and the equation simplifies to

$$
M_{x}=I_{x} \dot{\omega}_{x} \text { or simply } \boldsymbol{M}=\boldsymbol{I} \dot{\boldsymbol{\omega}}
$$

This equation will be the basis forming our equation of motion. Expanding the equation:

$$
M=\text { net moment }=\sum(\text { Gravitational Forces } X \text { Moment Arms })=\ddot{\phi} \sum_{j}^{3} m_{j} R_{j}^{2}
$$

Where $\mathrm{R}$ is the distance between satellite $\mathrm{CM}$ and the element $\mathrm{j}$. Element $\mathrm{j}$ can be either of the two stations or the tether. The word element will be used to describe an arbitrary part of the satellite.

Now, the distances between the stations' CM and Earth center can be found. Then the distance between the tether CM and the earth center can be found. These processes are described in the equations below:

$$
\begin{gathered}
r^{O \rightarrow \text { station } 1}=r \hat{a}_{1}-(d 0+d 1) \hat{b}_{1} \\
r^{O \rightarrow \text { station } 2}=r \hat{a}_{1}+(d 2+d 3) \hat{b}_{1} \\
r^{O \rightarrow \text { tetherCenter }}=r \hat{a}_{1}+\left(\frac{L}{2}-d 1\right) \hat{b}_{1}
\end{gathered}
$$

${ }^{3}$ See Nomenclature at end of paper 
Drawing a and $b$ reference frames together:

$$
\underbrace{\stackrel{\hat{a}_{2} \hat{b}_{1}}{\hat{b}_{1}}}_{\hat{a}_{1}} \quad \hat{a}_{1}=\hat{b}_{1} \cos (\phi)-\hat{b}_{2} \sin (\phi)
$$

Figure 4. Reference frames. Conveys the relationship between the intermediate and satellite reference frames

$$
\begin{gathered}
r^{O \rightarrow \text { station } 1}=r\left(\hat{b}_{1} \cos (\phi)-\hat{b}_{2} \sin (\phi)\right)-(d 0+d 1) \hat{b}_{1} \\
r^{O \rightarrow \text { station } 2}=r\left(\hat{b}_{1} \cos (\phi)-\hat{b}_{2} \sin (\phi)\right)+(d 2+d 3) \hat{b}_{1} \\
r^{O \rightarrow \text { tetherCenter }}=r\left(\hat{b}_{1} \cos (\phi)-\hat{b}_{2} \sin (\phi)\right)+\left(\frac{L}{2}-d 1\right) \hat{b}_{1} \\
\left|r^{O \rightarrow \text { station } 1}\right|=\sqrt{[r \cos (\phi)-(d 0+d 1)]^{2}+r^{2} \sin ^{2} \phi} \\
\left|r^{O \rightarrow \text { station } 2}\right|=\sqrt{[r \cos (\phi)+(d 2+d 3)]^{2}+r^{2} \sin ^{2} \phi} \\
\left|r^{O \rightarrow \text { tetherCenter }}\right|=\sqrt{[r \cos (\phi)+(d 1-(L / 2))]^{2}+r^{2} \sin ^{2} \phi}
\end{gathered}
$$

The equation for gravitational force is:

$$
\text { Force on an element }=\left(G M_{e} m_{\text {element }}\right) /\left(r^{O \rightarrow \text { element }}\right)^{2}
$$

We can define the direction of the force on an element as given below:

$$
\text { Direction of force on an element }=-r^{O \rightarrow \text { element }} /\left|r^{O \rightarrow \text { element }}\right|
$$


For simplicity, let station 1 be represented by s1, and station 2 by s 2 and tether $\mathrm{CM}$ as $\mathrm{t}$.

$$
\begin{gathered}
{\left[\frac{G M_{e} m_{1}}{\left(r^{O \rightarrow s 1}\right)^{2}} \cdot\left(\frac{-r^{O \rightarrow s 1}}{\left|r^{O \rightarrow s 1}\right|}\right) \times(-(\mathrm{d} 1+\mathrm{d} 0)) \hat{b}_{1}\right]+\left[\frac{G M_{e} m_{2}}{\left(r^{O \rightarrow s 2}\right)^{2}} \cdot\left(\frac{-r^{O \rightarrow s 2}}{\left|r^{O \rightarrow s 2}\right|}\right) \times(\mathrm{d} 2+\mathrm{d} 3) \hat{b}_{1}\right]} \\
+\left[\frac{G M_{e} m_{t}}{\left(r^{O \rightarrow t}\right)^{2}} \cdot\left(\frac{-r^{O \rightarrow t}}{\left|r^{O \rightarrow t}\right|}\right) \times((\mathrm{L} / 2)-d 1) \hat{b}_{1}\right] \\
=\ddot{\phi}\left[m_{1}(d 0+d 1)^{2}+m_{2}(d 2+d 3)^{2}+m_{t}((L / 2)-d 1)\right]
\end{gathered}
$$

The equation above is the general equation of motion for the unknown $\phi$. The expanded form is too long to be shown within the paper, but the breakdown of every term is given in the previous page. Using this, the EOM can be easily typed into MATLAB's ode45 function after a state space representation is performed. To simplify the state space representation, let:

$$
\begin{gathered}
M=\left[\frac{G M_{e} m_{1}}{\left(r^{O \rightarrow s 1}\right)^{2}} \cdot\left(\frac{-r^{O \rightarrow s 1}}{\left|r^{O \rightarrow s 1}\right|}\right) \times(-(\mathrm{d} 1+\mathrm{d} 0)) \hat{b}_{1}\right]+\left[\frac{G M_{e} m_{2}}{\left(r^{O \rightarrow s 2}\right)^{2}} \cdot\left(\frac{-r^{O \rightarrow s 2}}{\left|r^{O \rightarrow s 2}\right|}\right) \times(\mathrm{d} 2+\mathrm{d} 3) \hat{b}_{1}\right] \\
+\left[\frac{G M_{e} m_{t}}{\left(r^{O \rightarrow t}\right)^{2}} \cdot\left(\frac{-r^{O \rightarrow t}}{\left|r^{O \rightarrow t}\right|}\right) \times((L / 2)-d 1) \hat{b}_{1}\right]
\end{gathered}
$$

And

$$
I=\left[m_{1}(d 0+d 1)^{2}+m_{2}(d 2+d 3)^{2}+m_{t}((L / 2)-d 1)^{2}\right]
$$

The state variable definition is as follows:

$$
x_{1}=\phi, x_{2}=\dot{\phi}
$$

Therefore,

$$
\begin{gathered}
\dot{x_{1}}=x_{2} \\
\dot{x_{2}}=M / I
\end{gathered}
$$

This can be directly inputted into MATLAB after expansion, and any remaining cross products can be computed using MATLAB's “cross" function. For the circular orbit, since r, the radius of orbit, is constant, the computation is fairly straight forward. However, for the elliptical orbit, $r$ varies with the angle from periapsis (true anomaly). A method to numerically analyze rotation in the elliptical orbit will be discussed later. 


\section{Test Case Parameters}

Relatively arbitrary values were chosen to test the equations of motion and the numerical propagation methods. However, a sanity check was performed to ensure the values were within reason. The lists below define the parameters in this investigation. ${ }^{4}$

\subsection{Asymmetrical Satellite}
1) $\mathrm{d} 0=10 \mathrm{~m}$
2) $\mathrm{d} 3=10 \mathrm{~m}$
3) $\mathrm{L}=10 \mathrm{~km}$
4) $\mathrm{m} 1=5500 \mathrm{~kg}$
5) $\mathrm{m} 2=1500 \mathrm{~kg}$
6) $\mathrm{mt}=900 \mathrm{~kg}$

\subsection{Symmetrical Satellite}
1) $\mathrm{d} 0=10 \mathrm{~m}$
2) $\mathrm{d} 3=10 \mathrm{~m}$
3) $\mathrm{L}=10 \mathrm{~km}$
4) $\mathrm{ml}=1500 \mathrm{~kg}$
5) $\mathrm{m} 2=1500 \mathrm{~kg}$
6) $\mathrm{mt}=900 \mathrm{~kg}$

\subsection{Circular Orbit}

1) Radius of orbit $=8.371 \mathrm{~km}$ (upper limit of LEO)

\subsection{Elliptical Orbit}

1) Eccentricity $=0.5$

2) Semi-major axis $=21113 \mathrm{~km}$

\footnotetext{
${ }^{4}$ See Nomenclature at end of paper
} 


\section{Results}

\subsection{Circular Orbit Results}

As stated in the objectives section, seven cases were investigated, five with zero initial angular velocity and two with varying initial angular velocities. Appendix $\mathrm{B}(1)$ contains the code used for this analysis.

\subsubsection{Zero initial rotational angular velocity}

Different initial rotation angles: 0, 30, 60 and 90 degree cases were tested for nonsymmetric satellites. Additionally, one of the 90 degree cases included a symmetrical satellite. For certain trivial cases, the plots are shown only in the appendix.

1) $\phi(0)=0$ degrees, Asymmetric satellite

When the satellite is oriented in the vertical axis relative to earth $(\phi=0)$ with no angular velocity, the satellite is observed to stay at that orientation throughout its circular orbit. In other words, no rotation occurs throughout the orbit. Since this is a relatively trivial case, the plots are given in Appendix A(1). Since the satellite does not rotate, angular velocity also stays at zero $(\dot{\phi}=0)$. Additionally, there are no moments faced by the satellite.

\section{2) $\phi(0)=30$ degrees, Asymmetric satellite}

Figure 5 below shows the oscillations that take place in this situation. The satellite rotation occurs as oscillations about the mean of 90.016 degrees and has an amplitude of 60 degrees. The amplitude does not decay with time and the oscillation continues indefinitely. Figure 6 shows the moment time history faced by the satellite. The moment oscillates about 0 $\mathrm{Nm}$ and has an amplitude of $127200 \mathrm{Nm}$. The moment oscillations are not trivially sinusoidal; it is more complex as seen in figure 6. 


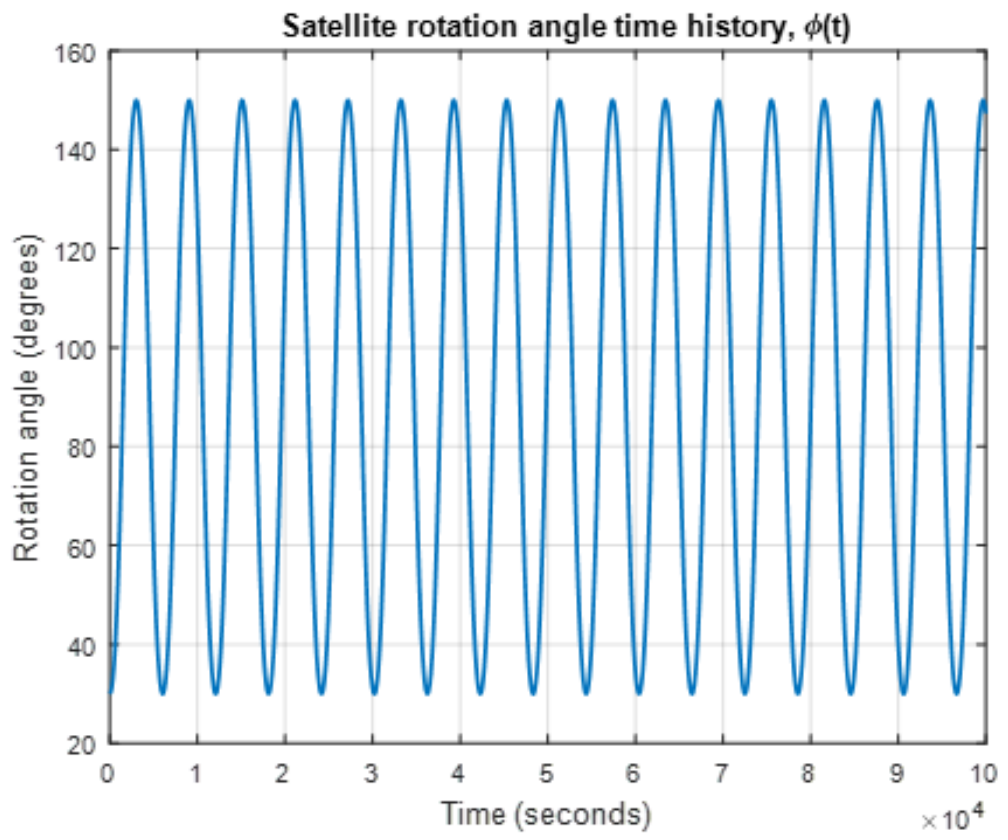

Figure 5. Angle time history. Shows the oscillating nature of the rotation angle for $\phi(0)=30$ degrees

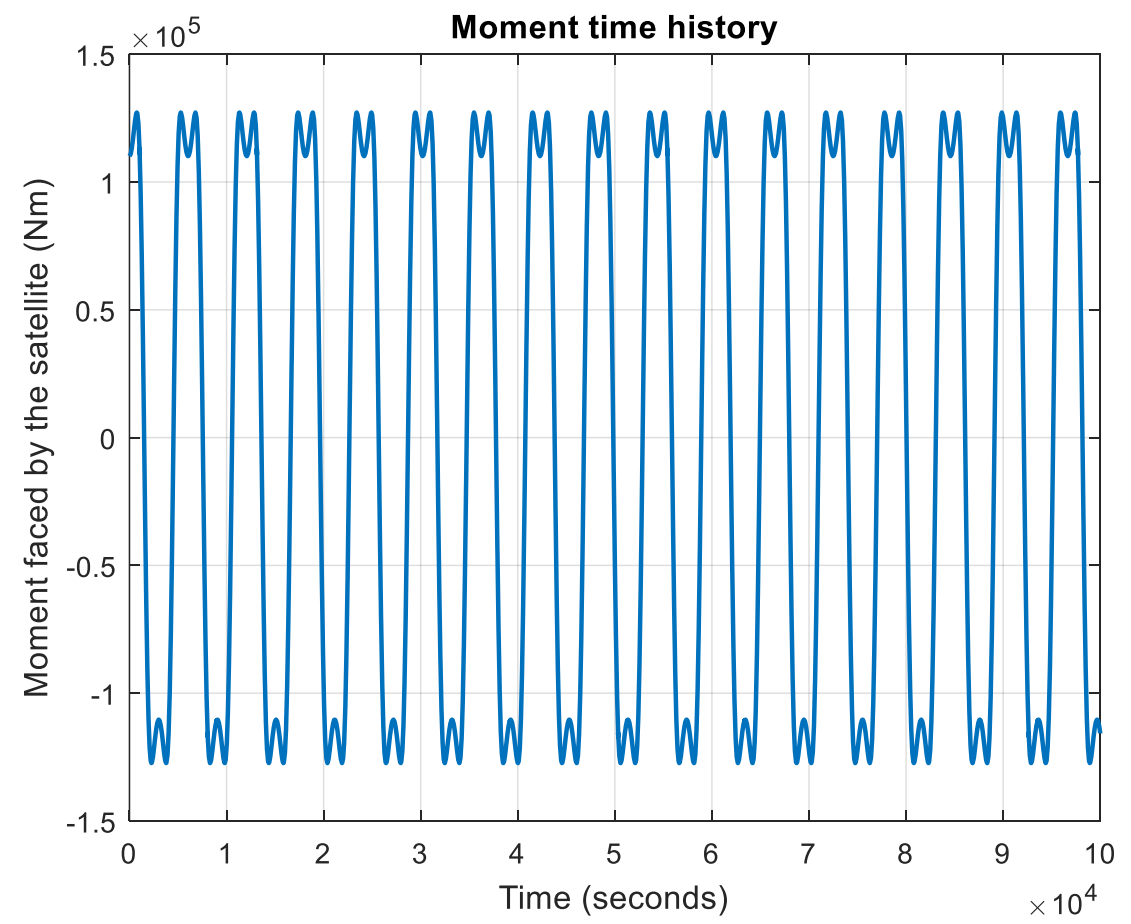

Figure 6. Moment time history. Shows the nontrivial oscillating nature of the moment for $\phi(0)=30$ degrees 
The angular velocity plot for this case is given in Appendix A(2). As expected, the angular velocity also oscillates about a set mean and has a non-decaying amplitude.

3) $\phi(0)=60$ degrees, Asymmetric satellite

The rotation angle oscillates about a mean (or equilibrium point) of 90.016 degrees but having a non-decaying amplitude of 30 degrees. This recurring equilibrium point which is slightly offset from 90 degrees is due to the asymmetry of the satellite. The amplitude of this case is analogous to the previous case when $\phi(0)=30$ degrees. A generalization can now be made for the amplitude of the oscillations for a non-zero initial rotational angle. The equation given below describes the relationship. Neglecting possible air/particle resistance and external forces other than gravity, the amplitude does not decay.

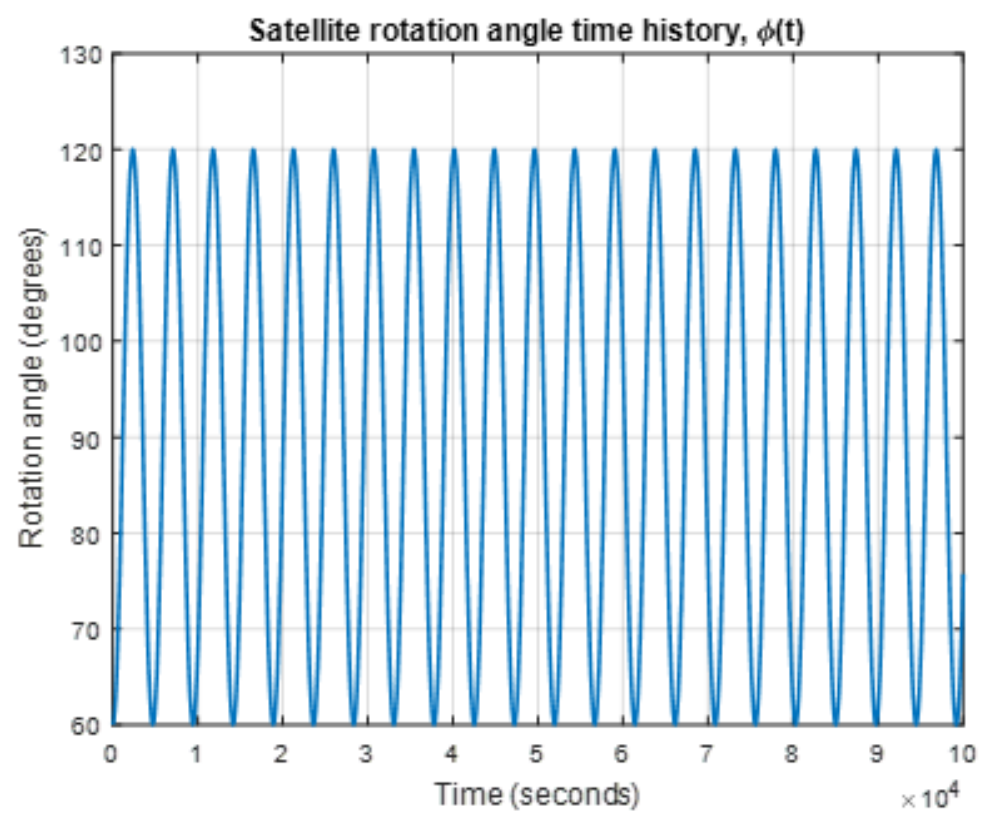

Figure 7. Rotation angle time history. Shows the oscillating nature of the rotation angle for $\phi(0)=60$ degrees

$$
\text { Amplitude }=\text { Equilibrium Angle }-\phi(0)
$$

Additional plots are given within Appendix A(3). As expected the angular velocity also oscillates as a derivative trend to the above plot. The moment oscillates in a more trivial fashion as opposed to the previous case. 
4) $\phi(0)=90$ degrees, Symmetric satellite

All the plots in this relatively trivial case is given within Appendix A(4). For a symmetric satellite, the equilibrium angle is 90 degrees. Therefore, using the Amplitude equation (24) given above, the Amplitude comes out to be 0 degrees. In other words, the rotation angle will remain 90 degrees throughout the orbit. The plots in A(4) show this case. Net moment comes out to be zero as seen in Appendix A(4).

5) $\phi(0)=90$ degrees, Asymmetric satellite

For an asymmetric satellite, the equilibrium point was determined to be 90.016 degrees. Therefore, using the Amplitude equation (24), the Amplitude was expected to be 0.016 degrees. And this was proven by the numerical result in Figure 8 shown below:

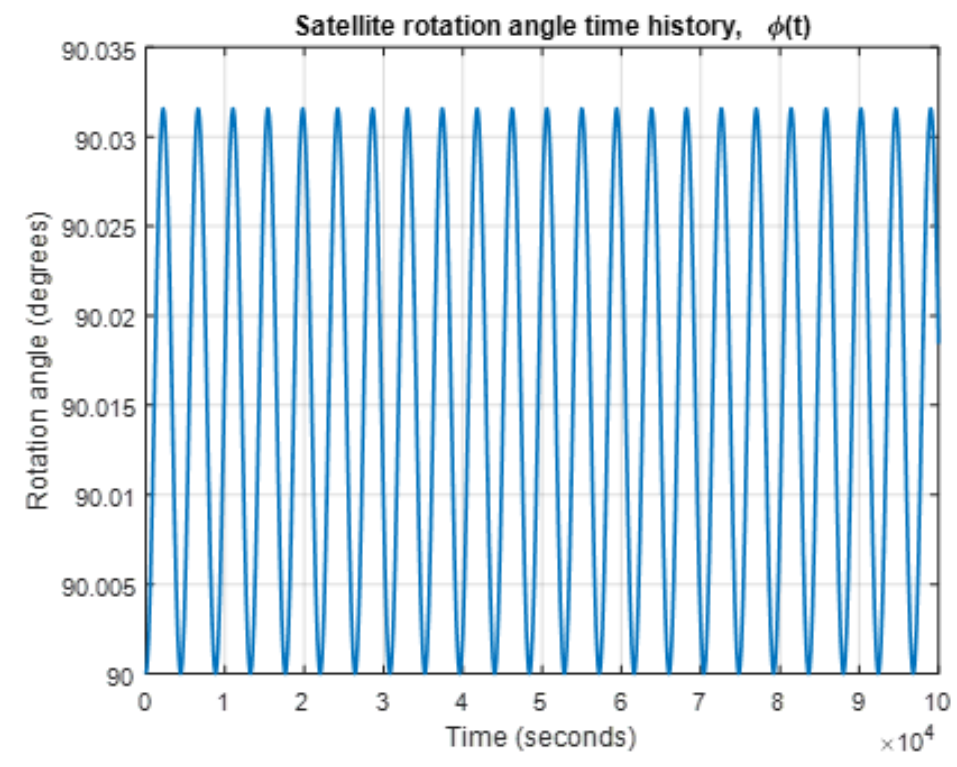

Figure 8. Angle time history. Shows the oscillating nature of the rotation angle for $\phi(0)=90$ degrees Asymmetric Satellite.

Additional plots are given in Appendix A(5). The value of the moments are small (with a max magnitude of $70 \mathrm{Nm}$ ) due to the fact that $\phi(0)$ was only slightly perturbed from the equilibrium angle of 90.016 degrees. 
B. Non-zero initial rotational angular velocity, $\dot{\phi}(0) \neq 0$ (With $\phi(0)$ constant at 60 degrees)

1) $\dot{\phi}(0)=0.01$ degrees/second, Asymmetric satellite

The rotations in this case (and for lower $\dot{\phi}(0)$ values) also occur as oscillations about 90.016 degrees as seen in Figure 9. However, the amplitude is greater than the difference between the equilibrium angle and $\phi(0)$ due to the non-zero initial angular velocity.

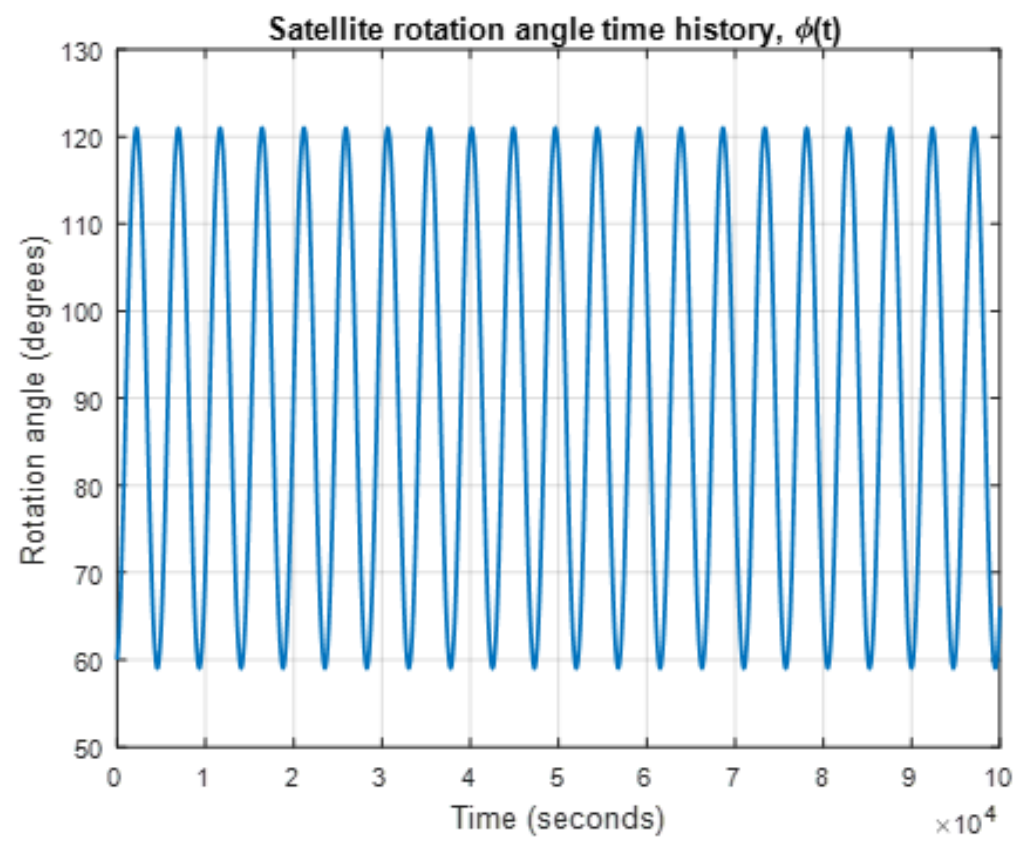

Figure 9. Angle time history. Shows the oscillating nature of the rotation angle for

$$
\dot{\phi}(0)=0.01 \text { degrees per second Asymmetric Satellite. }
$$

The angular velocity and moment also have oscillatory trends as seen in previous cases. The next case will involve an initial angular velocity that has been increased by a magnitude of ten.

2) $\dot{\phi}(0)=0.10$ degrees/second, Asymmetric satellite

This case involves a much greater initial angular velocity, one that causes the satellite to stop having oscillatory rotations and to spin. As seen in Figure 10, the satellite now begins to spin without an equilibrium point. In other words, the quantity $\phi$ continually increases. Despite the appearance in Figure 10, the line is not straight and has some fluctuations. This is due to the oscillating angular velocity shown in figure 11. 


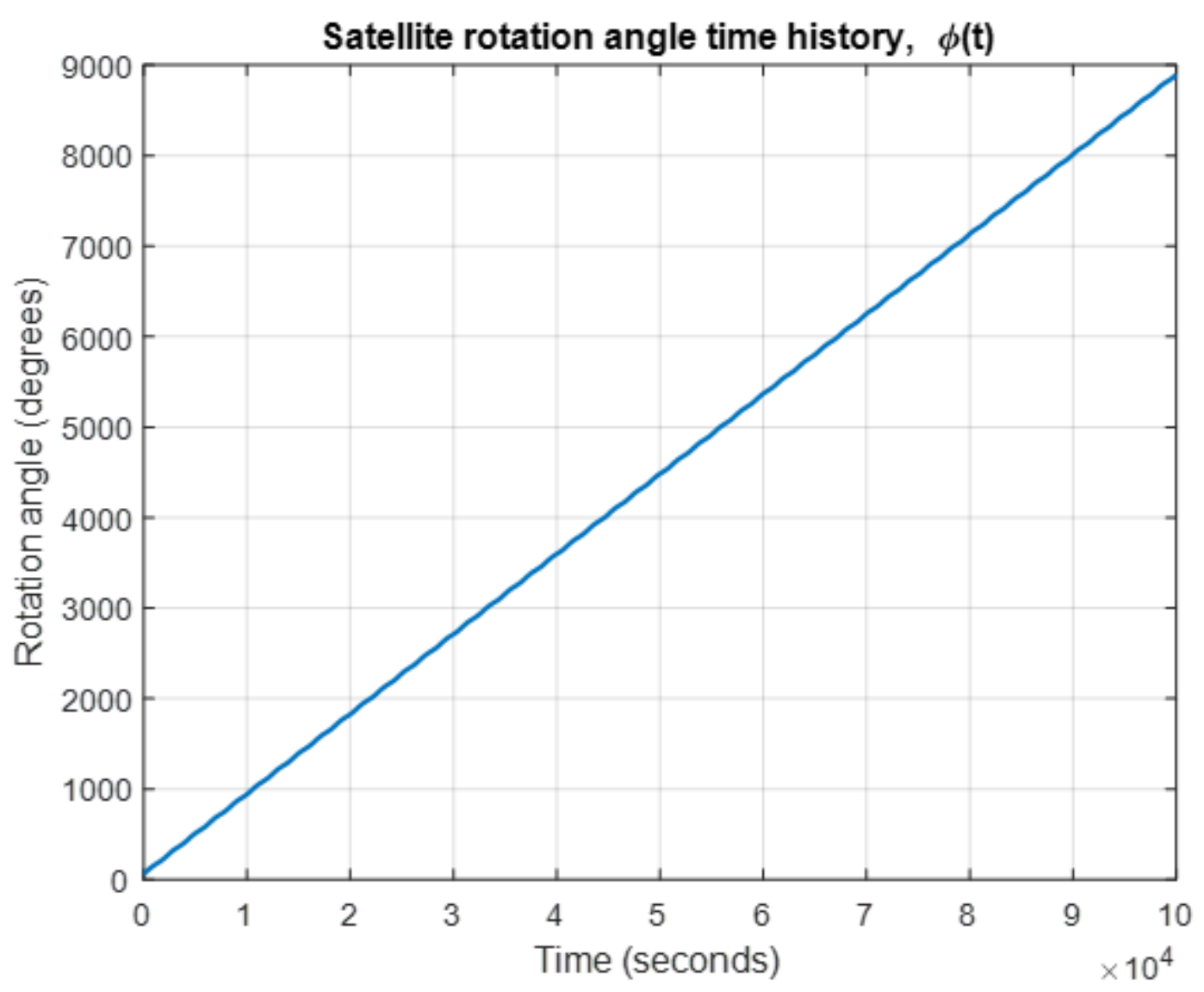

Figure 10. Angle time history. Shows the increasing rotation angle for $\dot{\phi}(0)=0.1$ degrees per second Asymmetric Satellite. (Spinning)

From Figure 11 in the next page, it can be seen that angular velocity oscillates between 0.071 to 1.08 degrees per second at a very high frequency. Since angular velocity stays positive, the direction of rotation does not change and no rotation angle oscillations take place. 


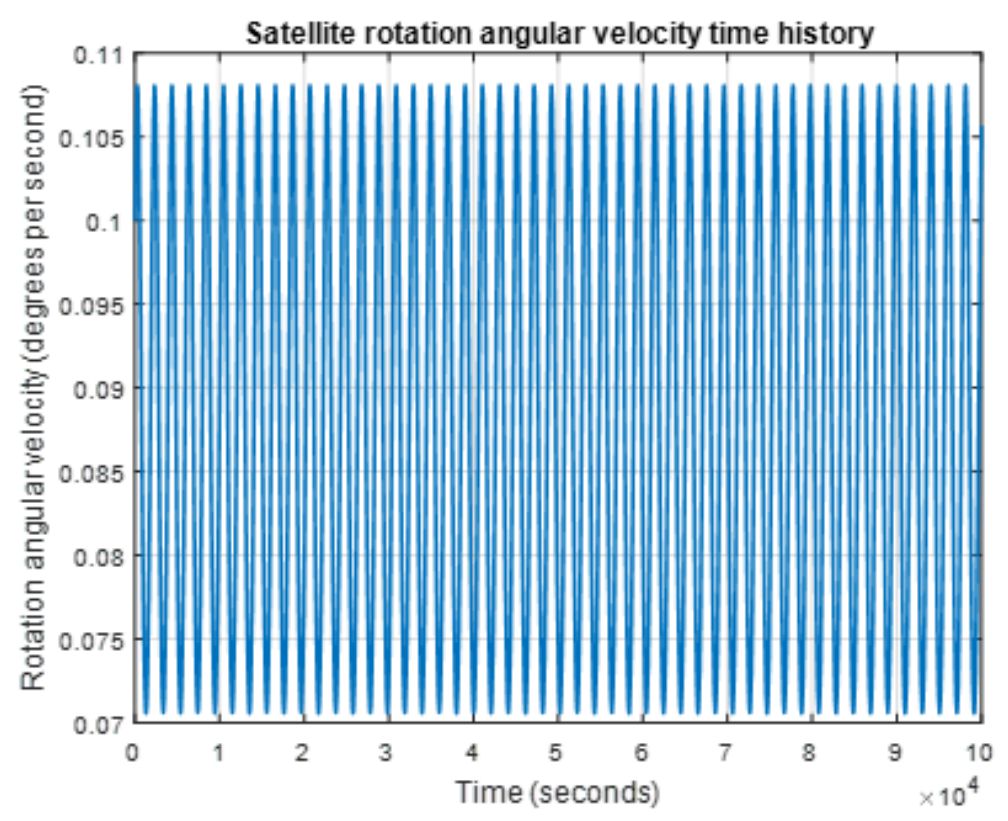

Figure 11. Angular Velocity time history. Shows the high frequency angular velocity for $\dot{\phi}(0)=0.1$ degrees per second Asymmetric Satellite. (Spinning)

Figure 12 shows the net moment faced by the satellite in the circular orbit. Because the satellite is spinning, the moment oscillates about $0 \mathrm{Nm}$ at a much higher frequency than in previous cases.

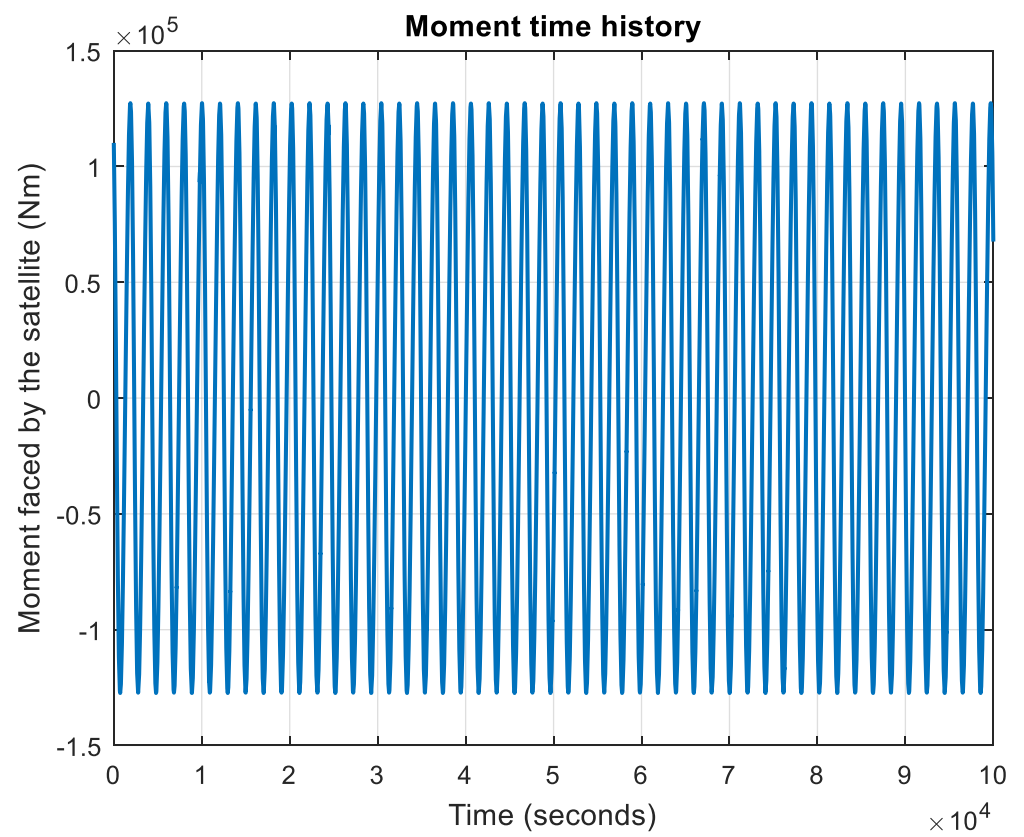

Figure 12 (left). Moment time history. Shows the high frequency net moment for $\dot{\phi}(0)=0.1$ degrees per second Asymmetric Satellite. (Spinning) 


\subsection{Elliptical Orbit Numerical Results}

\subsubsection{Elliptical Orbit Method Introduction}

For the elliptical orbit, the radius of orbit changes with the orbit angle, $\theta$. The complexity for the elliptical orbit lies in the fact that the orbit angle $\theta$, is not an explicit function of time. Therefore in ODE function being iterated, the value of $\theta$ cannot be found at different times. However, one work around was to get time in terms of $\theta$. Therefore, in a separate function, varying theta from 0 to 360 degrees, values of times can be calculated at which each orbit angle would occur. This array of time would then be inputted as the "time span" field of the ode45 function in MATLAB. Within the ODE function being iterated, the orbit angle can now be found by relating the $\theta$ array to the time array. Once $\theta$ is known, $r$ can be found and the EOM derived can be used.

According to the fundamentals of astrodynamics, the set of equations stated below will be used to relate $\mathrm{r}$ to $\theta$ and $\theta$ to time.

$$
\begin{gathered}
r=\frac{a\left(1-e^{2}\right)}{1+e \cos (\theta)} \\
\cos (E)=\frac{e+\cos (\theta)}{1+e \cos (\theta)} \\
\text { MeanAnomaly }=E-e \sin (E) \\
t=\left(\frac{a^{3}}{k}\right)^{0.5} \cdot \text { MeanAnomaly }
\end{gathered}
$$

Appendix $\mathrm{B}(2)$ contains the codes used for this investigation. Figure 13 shows the orbit angle time history $\theta(\mathrm{t})$ for one revolution around earth with $\mathrm{e}=0.5$, and $\mathrm{a}=2111.3 \mathrm{~km}$. 


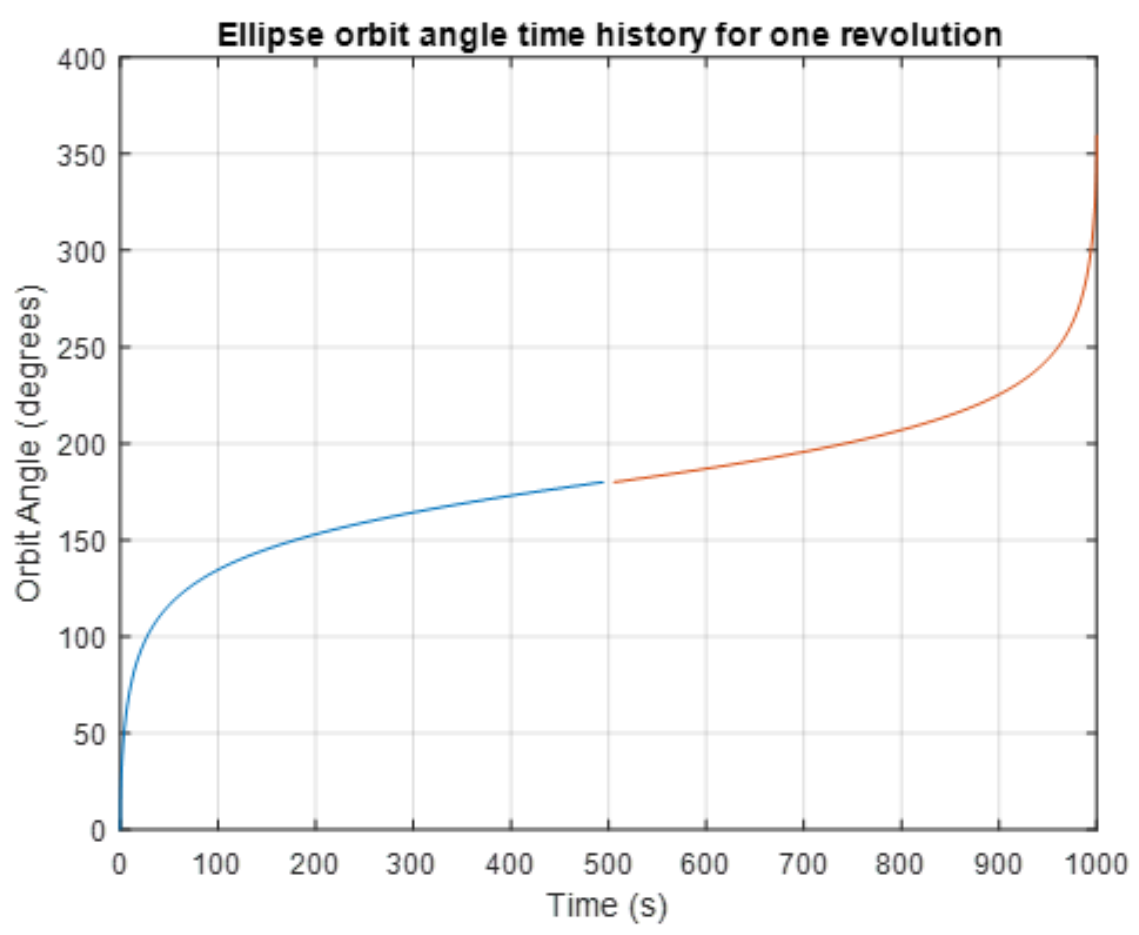

Figure 13. Orbit angle time history. Shows the orbit angle time history for the elliptical orbit.

The figure above shows one revolution; 10 of these revolutions are performed in the following numerical analysis. With this method, an initial rotation angle and angular velocity can be specified and the program will perform the numerical analysis to estimate rotation angle time history. The results of the numerical analysis with a variety of initial condition choices show that an elliptical orbit is a lot more complex to generalize into a trend. No specific oscillations or patterns occur other than the fact that $\dot{\phi}(0)=0.05$ or greater would cause constant one directional spinning as seen previously with high initial angular velocities. Other than the spinning observed for high initial angular velocities, the results were more complex. For example, for $\phi(0)=30$ degrees and $\dot{\phi}(0)=0$, Figure 14 shows fluctuation in angle of rotation but with no specific amplitude or mean. 


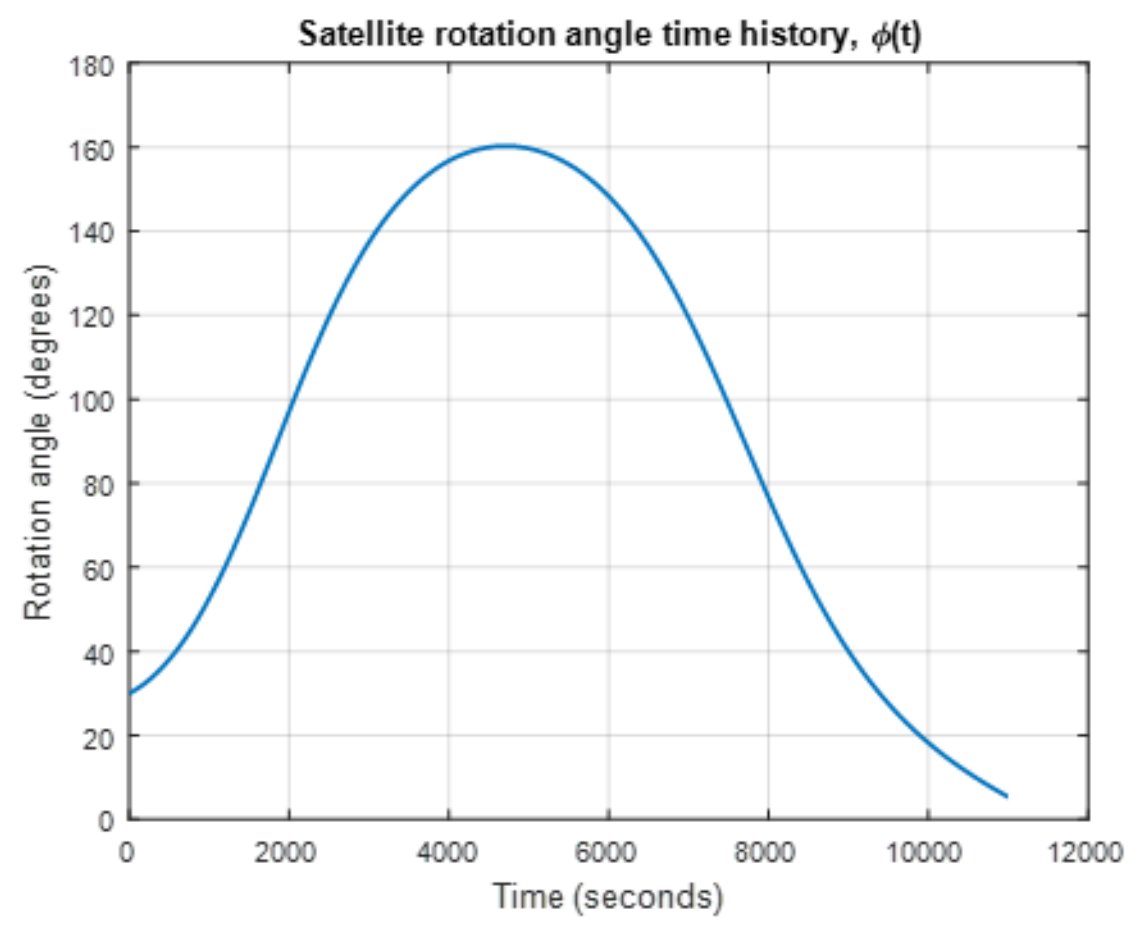

Figure 14. Orbit angle time history. Shows the rotation angle time history for the elliptical orbit $\phi(0)=30$ degrees and $\dot{\phi}(0)=0$. No notable pattern or specific oscillation

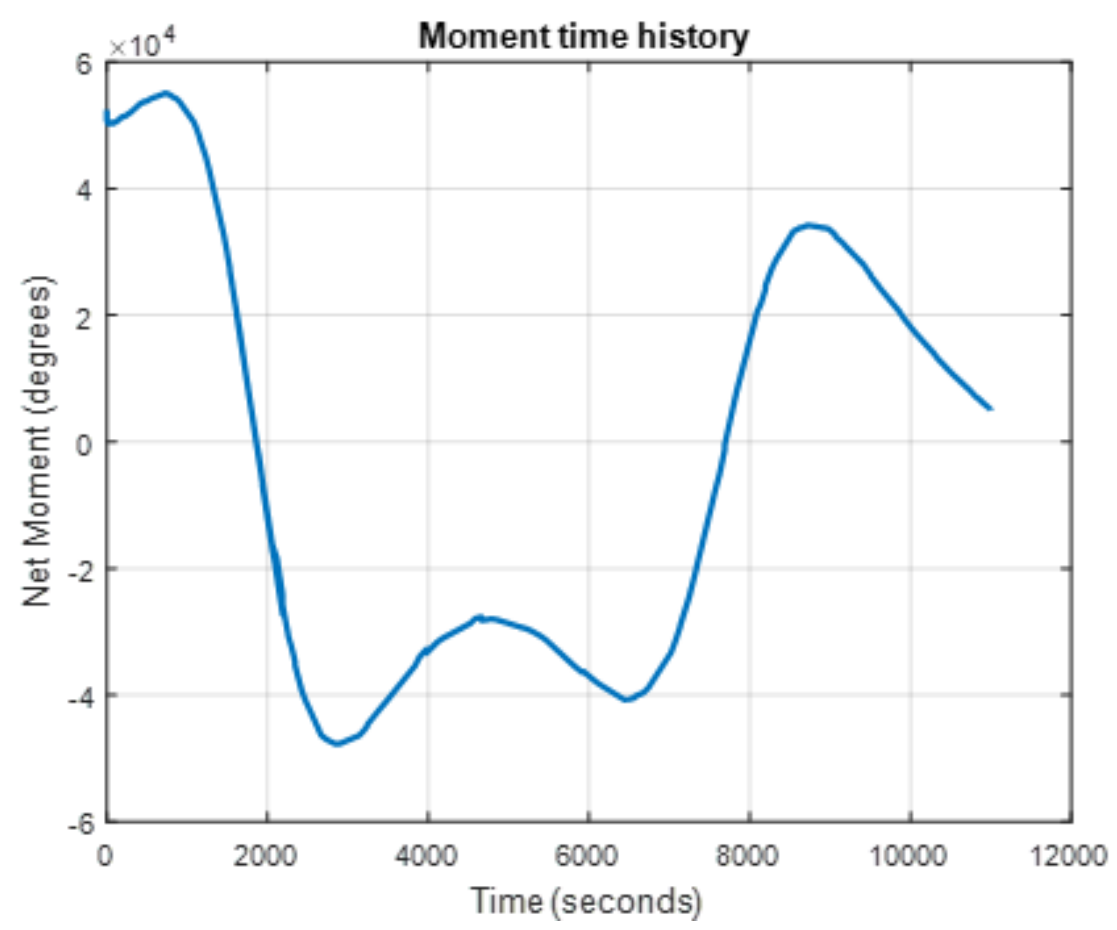

Figure 15. Net Moment time history. Shows the Net Moment time history for the elliptical orbit $\phi(0)=30$ degrees and $\dot{\phi}(0)=0$. No notable pattern or oscillations. 


\section{Conclusion}

For circular orbit, specific patterns were found for the cases investigated. However, for the elliptical orbit, specific patterns were not found. The conclusions from this investigation are listed below:

\subsection{Circular Orbit}

1) With zero initial angular velocity $\dot{\phi}(0)=0$ and $\phi(0)=0$, the satellite remains at $\phi(0)$ $=0$ indefinitely.

2) With zero initial angular velocity $\dot{\phi}(0)=0$ and $\phi(0) \neq 0$, the satellite will oscillate about its equilibrium angle (around 90 degrees) indefinitely with a constant Amplitude equal to the difference between the equilibrium angle and the initial rotation angle $\phi(0)$.

a. This implies that $\phi=0$ is an unstable equilibrium.

3) The equilibrium angle is 90 degrees for a symmetric satellite but slightly different for an Asymmetric satellite (90.016 degrees for the asymmetric satellite in this paper).

4) A small angular velocity will cause the satellite to have an amplitude that is greater than the difference between the equilibrium (or mean) angle and the initial rotation angle $\phi(0)$.

5) A large angular velocity will result in a constant, unidirectional spin, leading to very high frequency moment oscillations; this could lead to fatigue in the tethered satellite structure.

a. For this asymmetric satellite in circular orbit, spinning occurs when

$$
\dot{\phi}(0)>0.075 \mathrm{deg} / \mathrm{s} \text {. }
$$

\subsection{Elliptical Orbit}

1) The paper outlines a method for numerically estimating the rotation angle of a tethered satellite in elliptic orbit. However, no specific or conclusive pattern was observed within the investigation.

2) A large angular velocity will result in a constant, unidirectional spin, leading to very high frequency moment oscillations; this could lead to fatigue in the tethered satellite structure. 
a. For this asymmetric satellite in elliptical orbit, spinning occurs when $\dot{\phi}(0)>0.043 \mathrm{deg} / \mathrm{s}$.

The numerical estimation given in this paper outlines time histories of the angular rotations which would help with docking missions to a futuristic tethered satellite. Certain limitations apply with the methods outlined in the paper.

1) Numerical integration will have increasing error with propagation of time, especially if the time step provided is large.

2) The region of orbit is assumed to be perfectly free of air/particle resistance

3) Further research should be done into the elliptical orbit methods in order to account for more revolutions and refine the current method.

However, overall the investigation introduces a new futuristic topic and ways it can be handled or studied. 


\section{Appendix}

\section{Appendix A. Nomenclature}

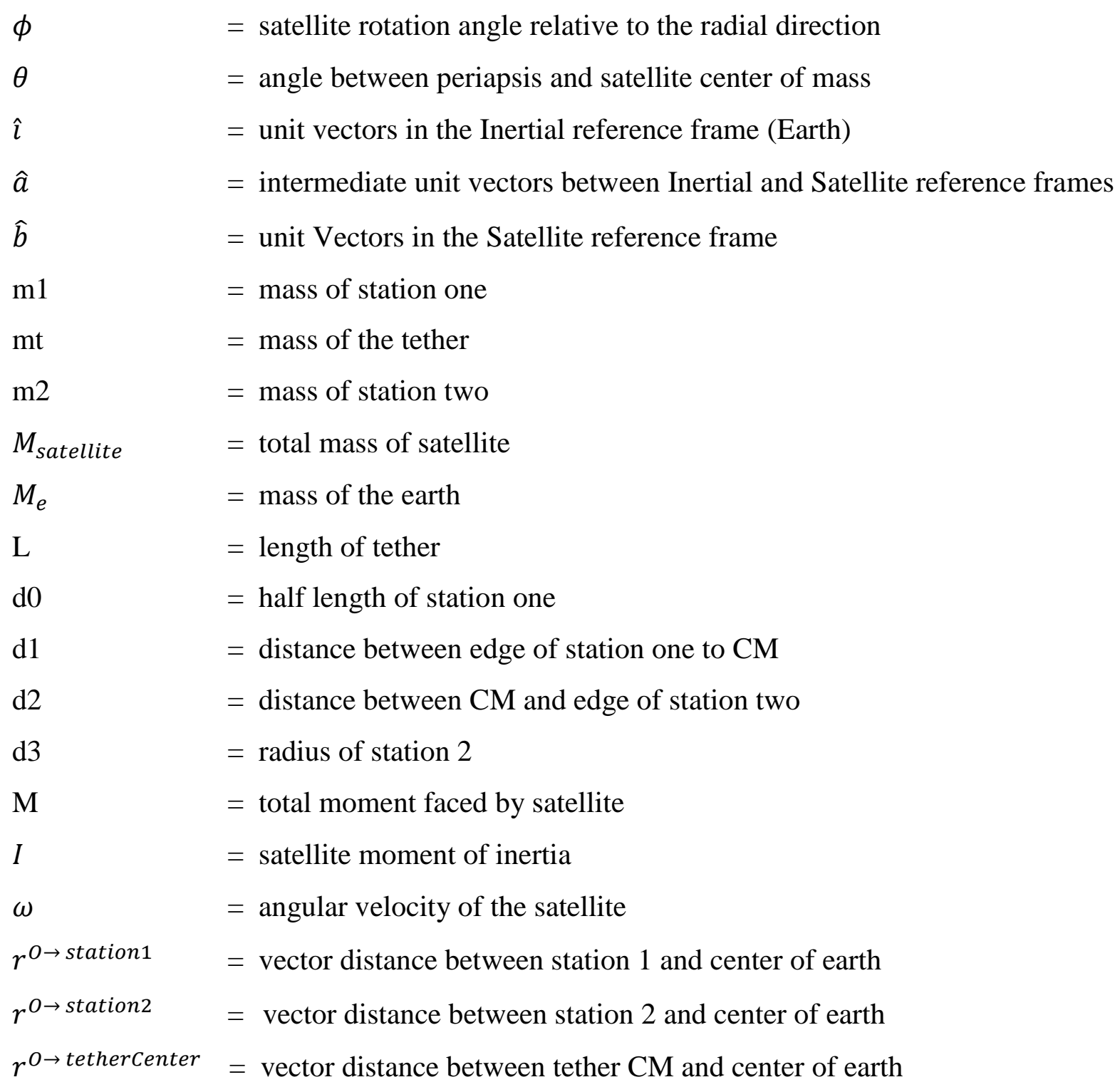


Appendix B. Excess plots/plots of relatively trivial cases $(\dot{\phi}(0)=0$ degrees per second)

1) $\phi(0)=0$ degrees, Asymmetric satellite
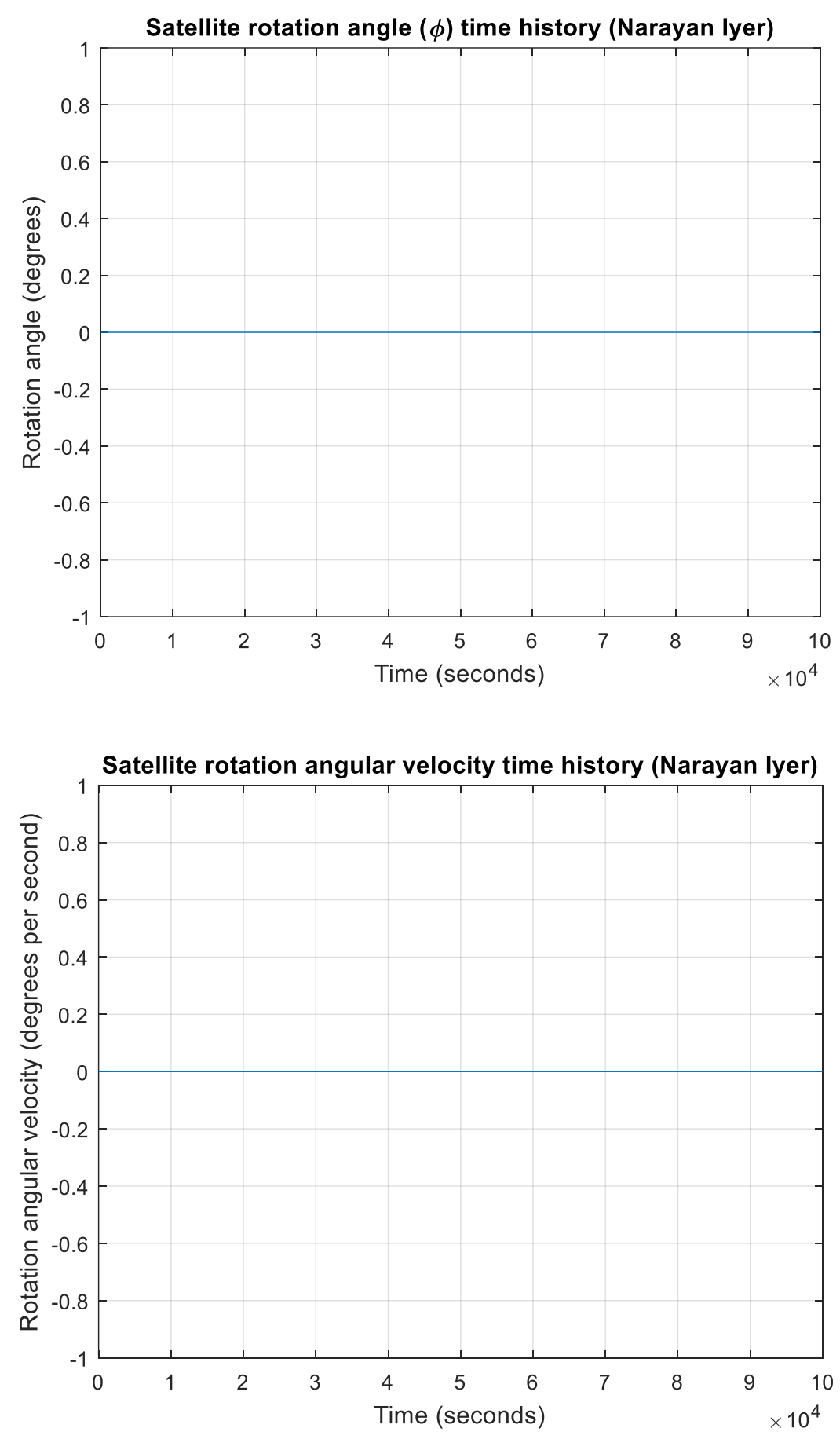


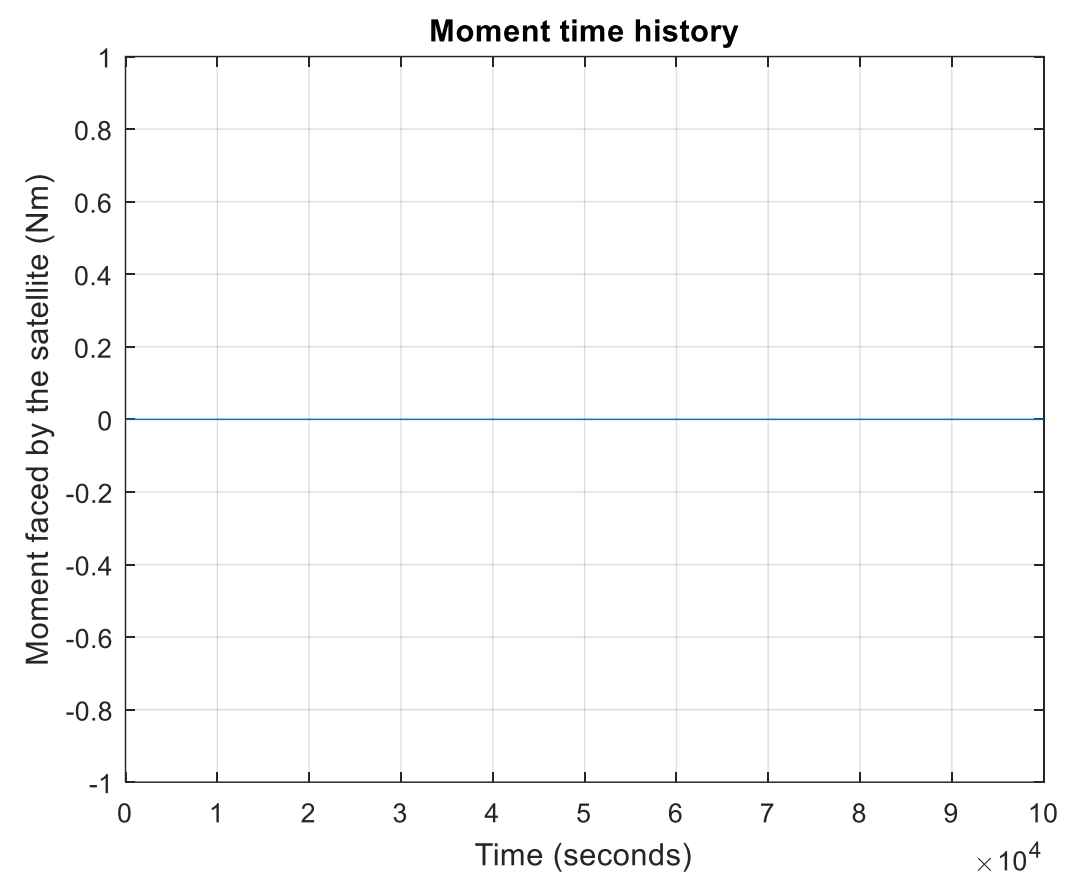

2) $\phi(0)=30$ degrees, Asymmetric satellite (Additional Plot)

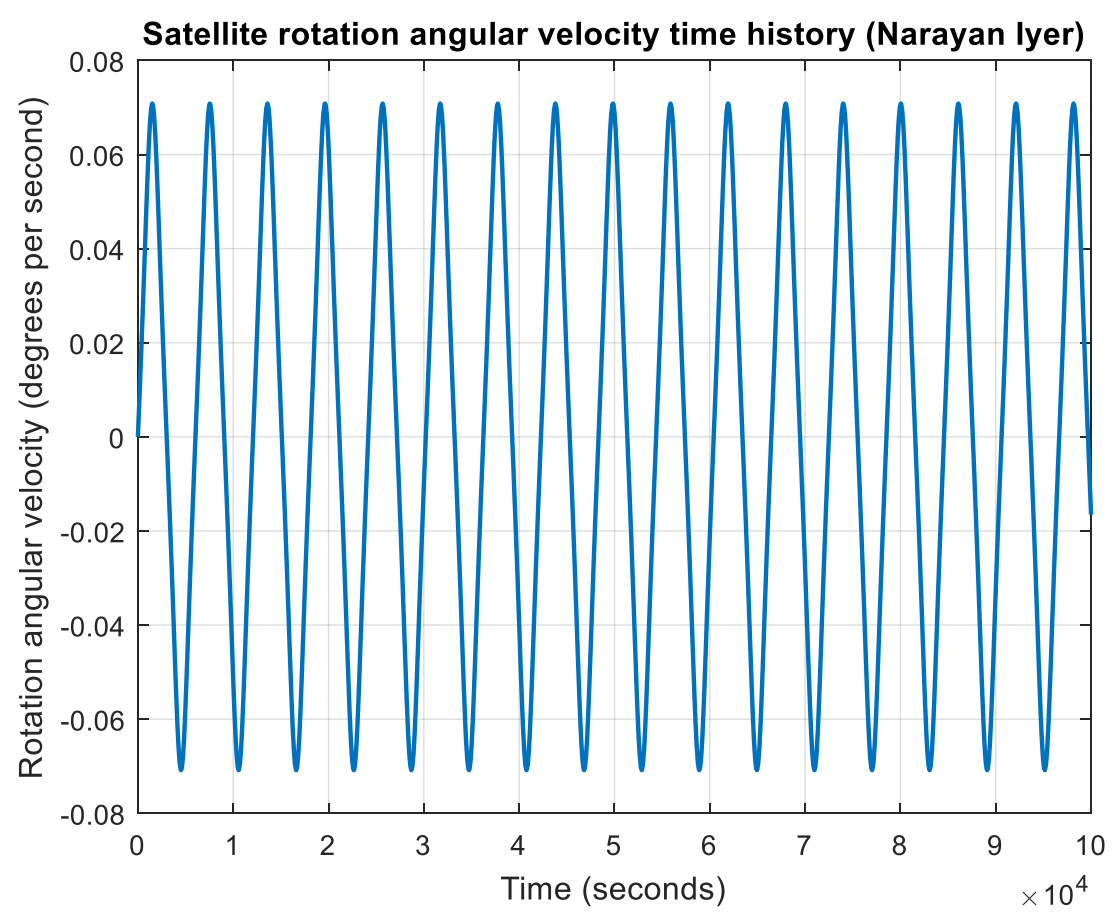


3) $\phi(0)=60$ degrees, Asymmetric satellite (Additional Plots)
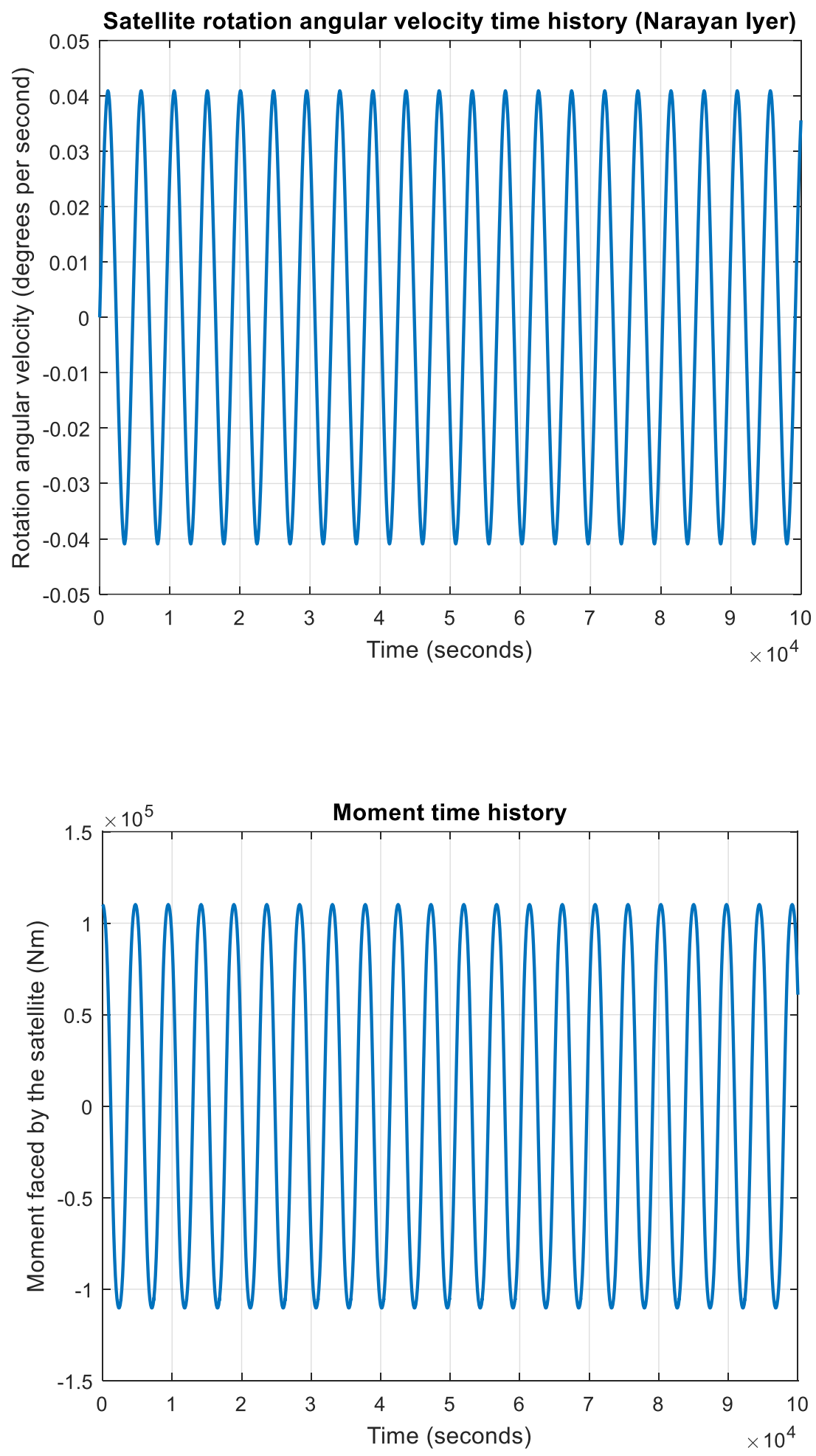
4) $\phi(0)=90$ degrees, Symmetric satellite (All Plots)
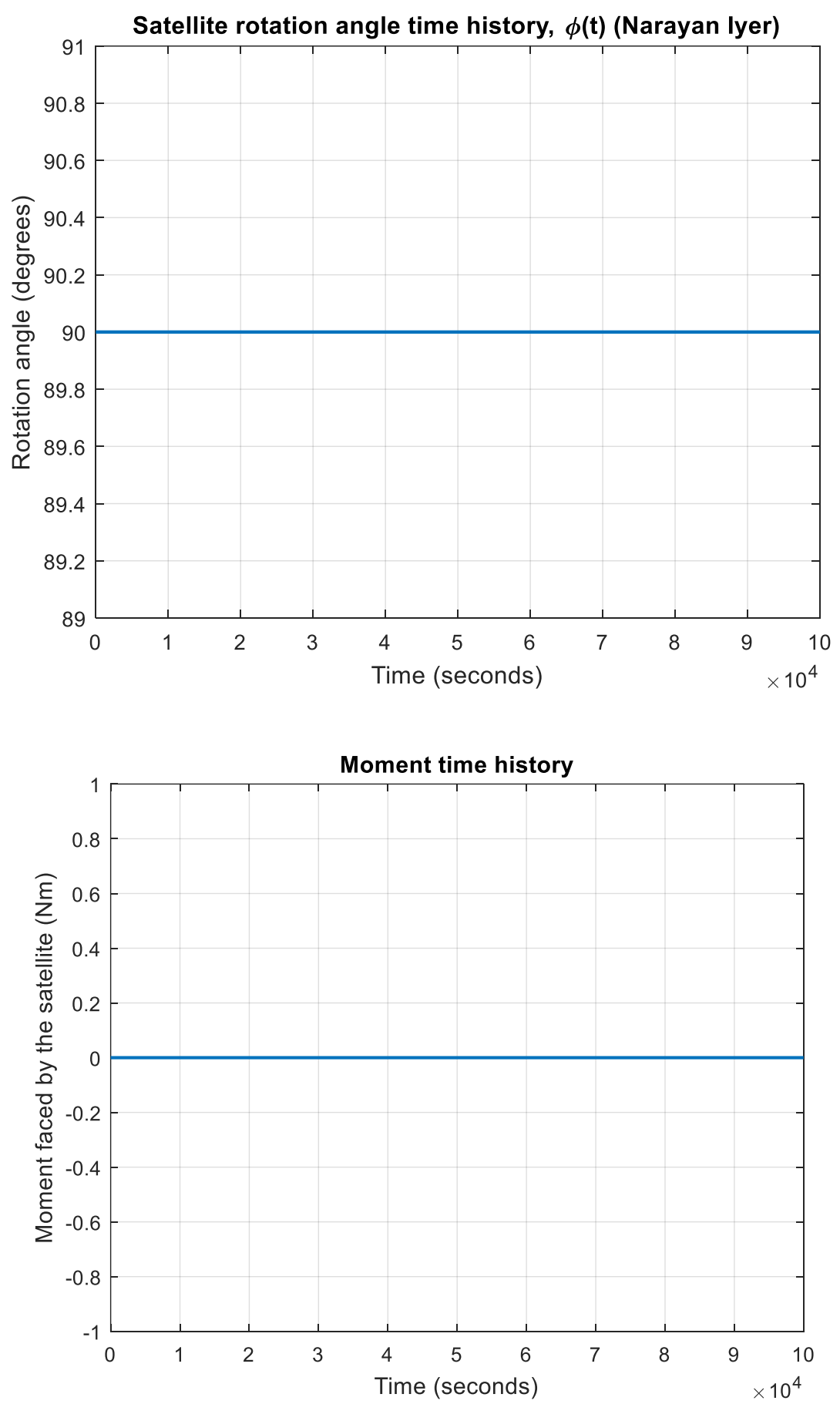
5) $\phi(0)=90$ degrees, Asymmetric satellite (Additional Plots)
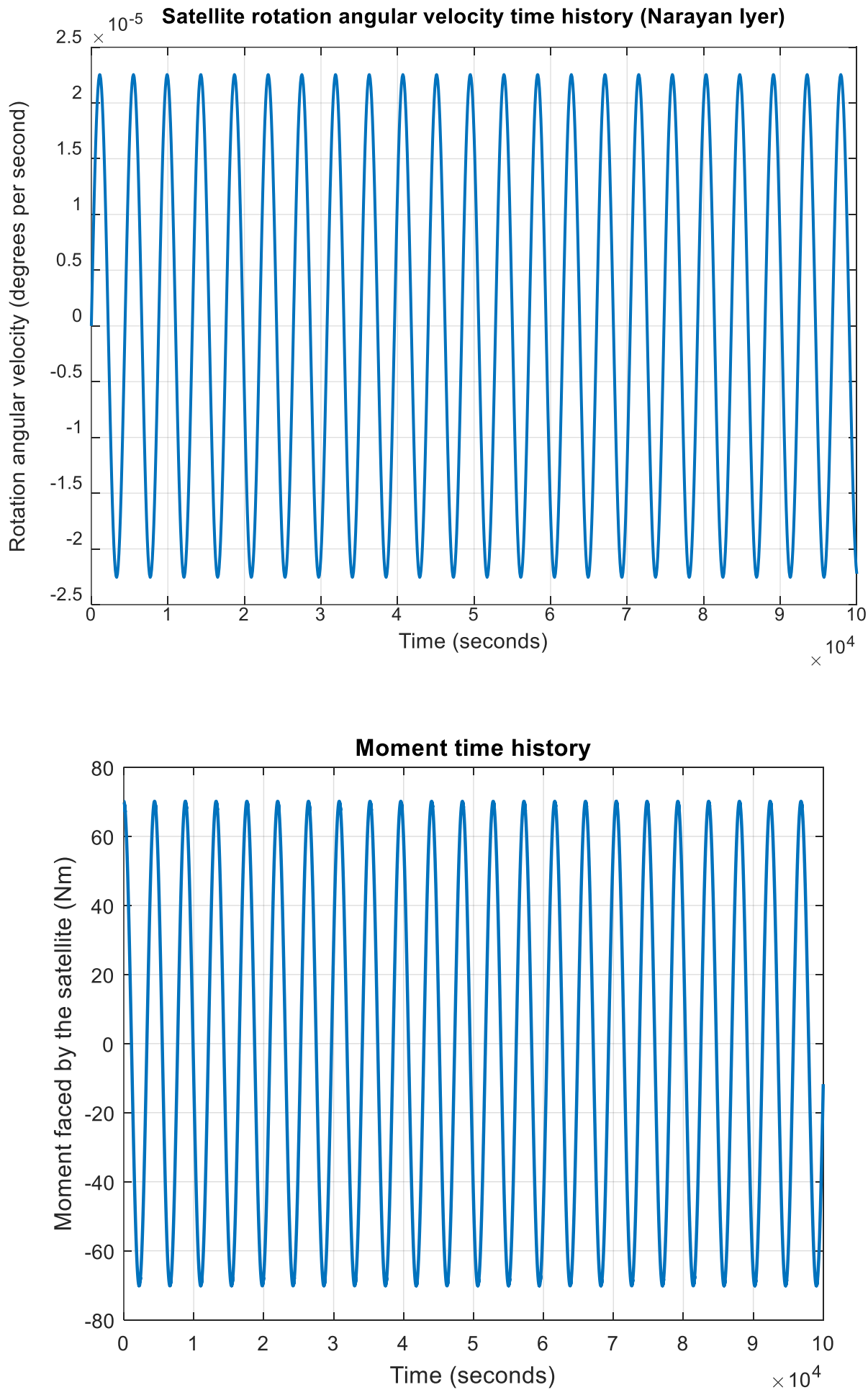


\section{Appendix C. Codes Used}

\section{1) Circular Orbit}

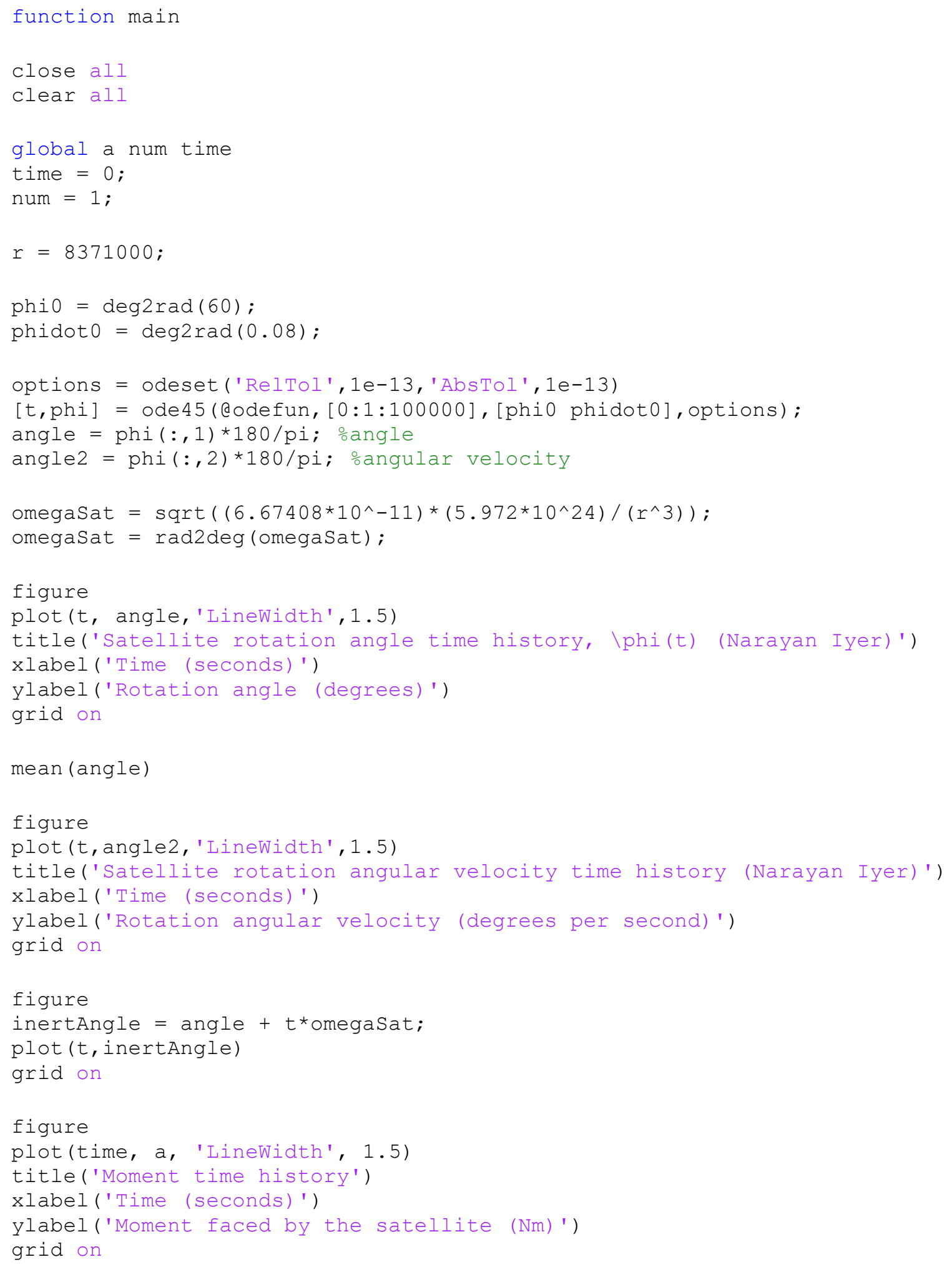




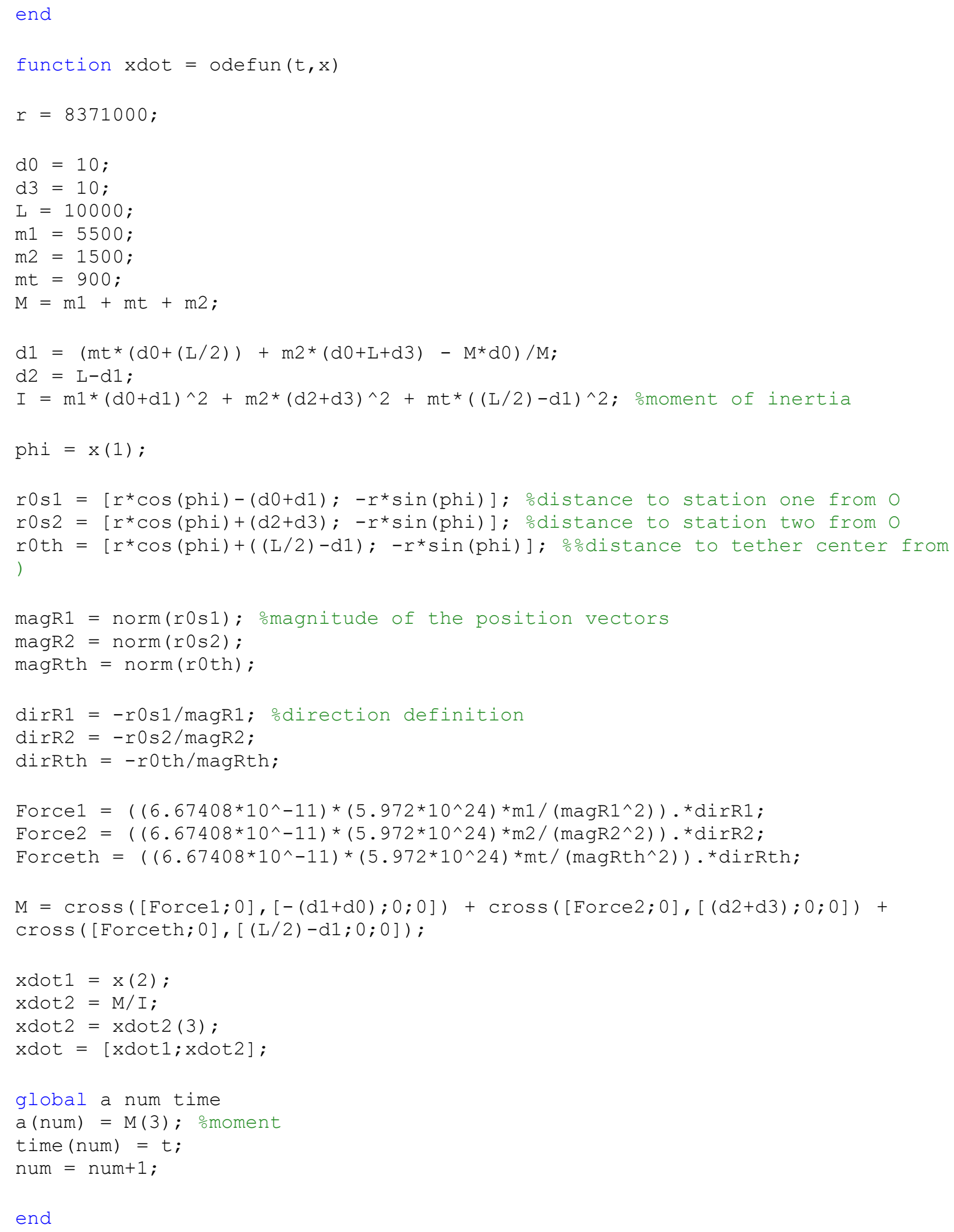




\section{2) Elliptical Orbit}

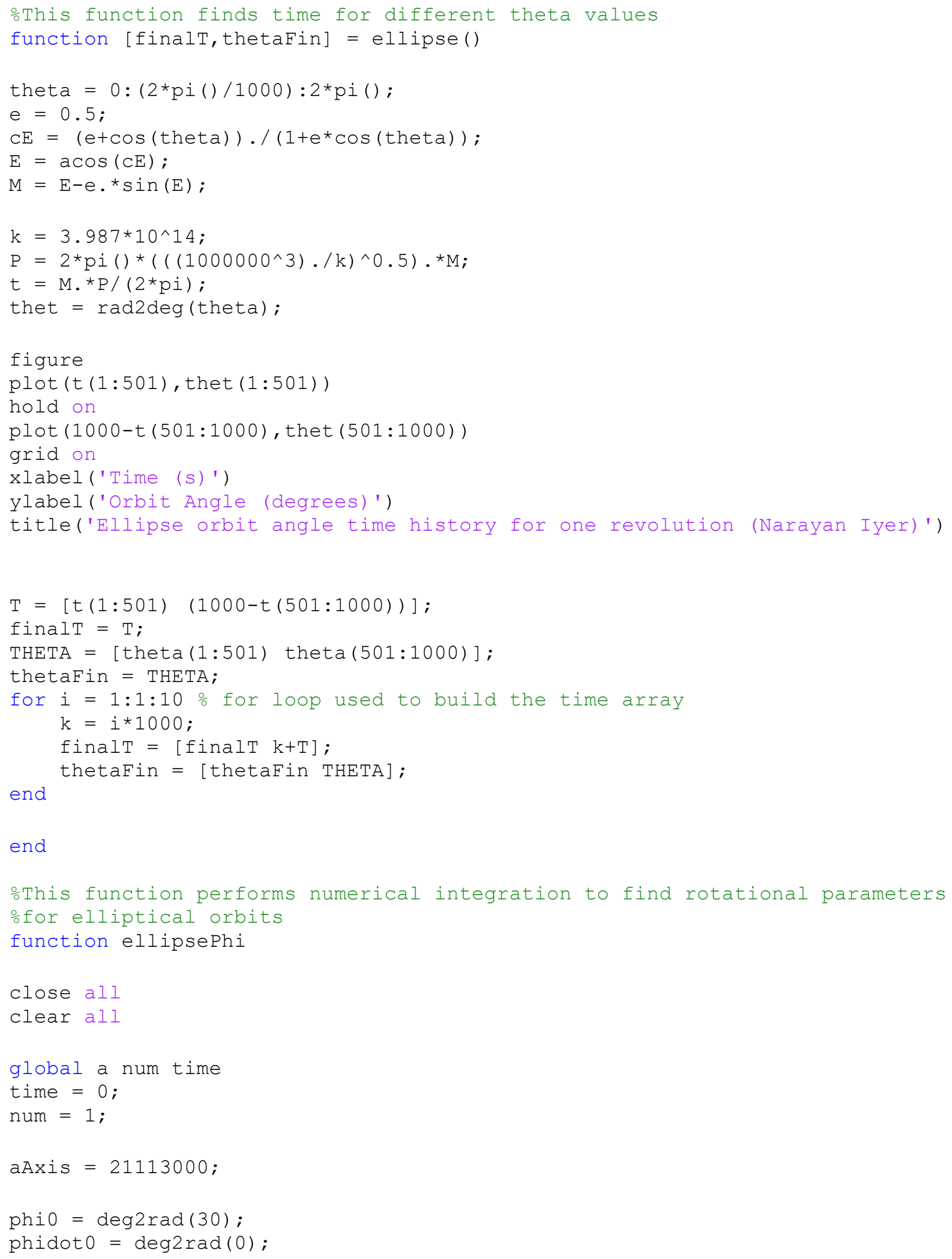




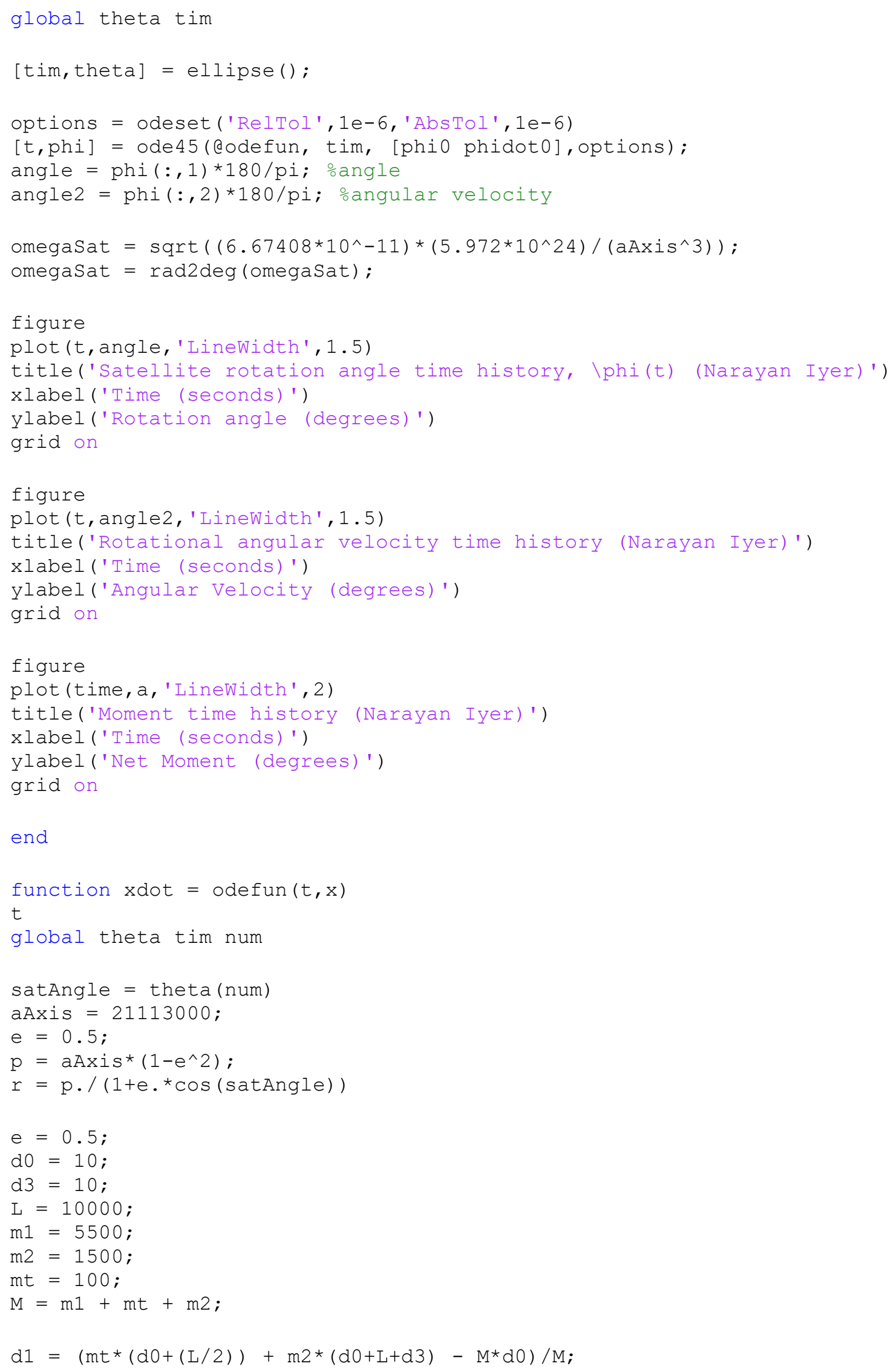




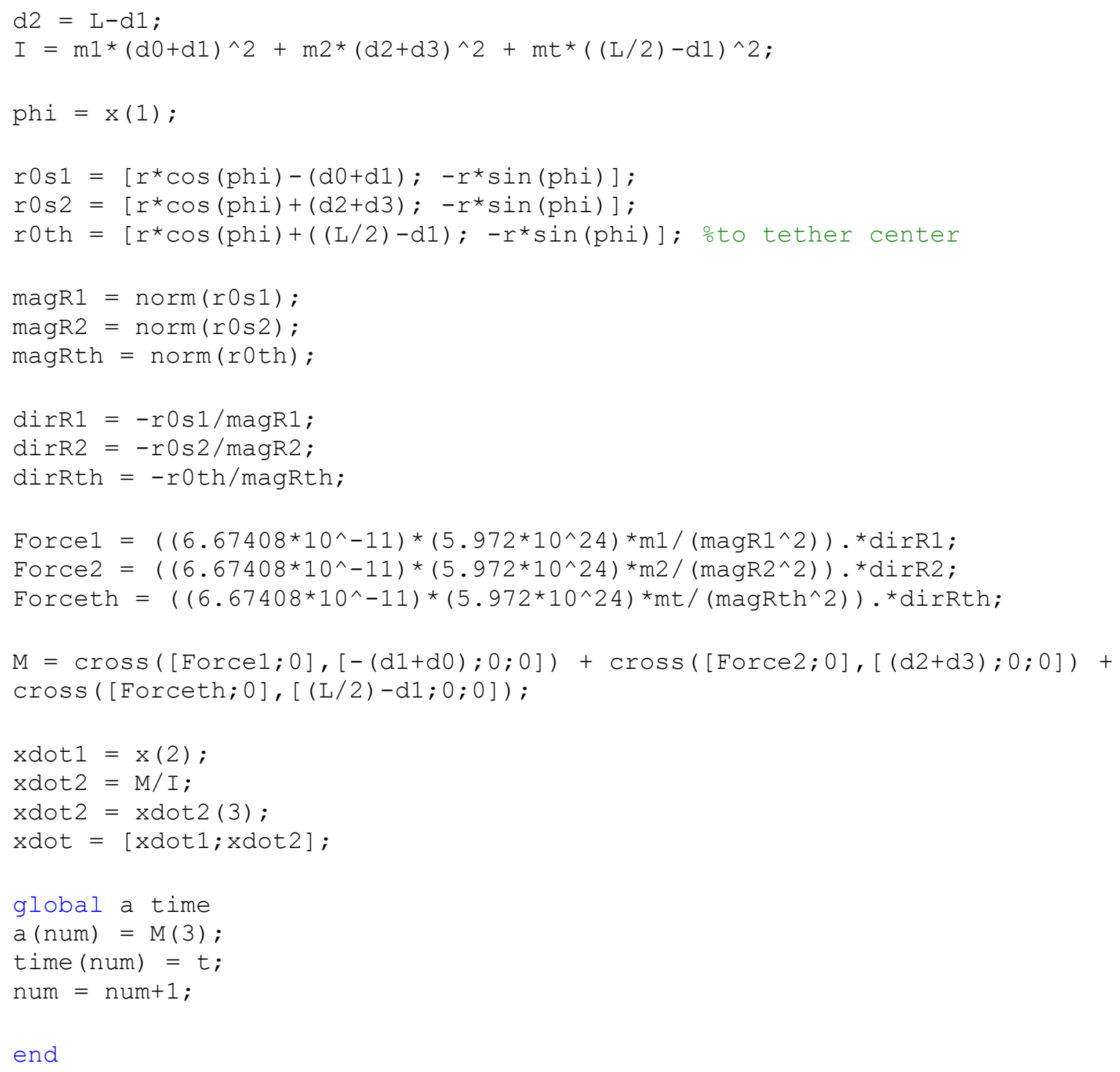




\section{References}

- Greenwood, D., Principles of dynamics, Ann Arbor, MI: 1987.

- Bate, R. R., Mueller, D. D., and White, J. E., Fundamentals of Astrodynamics, White Dover Publications, 1971.

- José J V, and Saletan, E. J., Classical dynamics: A Contemporary Approach, Cambridge: Cambridge University Press, 1998.

- Sanyal, A. K., Shen, J., and McClamroch, H. N., "Dynamics and Control of an Elastic Dumbbell Spacecraft in a Central Gravitational Field."

- Tsiotras, P., and Longuski, J. M., “Analytic Solutions for a Spinning Rigid Body Subject to Time Varying Body-Fixed Torques, Part II: Time-Varying Axial Torque ," vol. 60, Dec. 1993, pp. 976-981.

- Anderson, J. D., Introduction to flight, New York: McGraw-Hill, 1985.

- Nave, R., "Rotational-Linear Parallels,” Available: http://hyperphysics.phyastr.gsu.edu/hbase/mi.html. 